\title{
UNICOHERENCIAS EN ESPACIOS SÓLIDOS
}

\author{
William C. Olano Díaz ${ }^{1}$
}

\begin{abstract}
RESUMEN.- Se da una implicación entre la "Solidez" y la «unicoherencia» en espacios normales y localmente conexos por caminos usando los métodos de la Topología Algebraica que consiste en asociar a cada espacio topológico $X$ un grupo $B(X)$ denominado "Grupo de Bruschlinsky».
\end{abstract}

\section{INTRODUCCIÓN}

En el presente trabajo se da una implicación entre la "solidez" y la "unicoherencia" en espacios normales y localmente conexos por caminos usando los métodos de la Topología Algebraica que consiste en asociar a cada espacio topológico $X$ un grupo $B(X)$ denominado "Grupo de Bruschlinsky".

Los espacios sólidos están intimamente relacionados con los retractos absolutos, este resultado tiene uso frecuente en problemas de "extensión y homotopías"; aún más para los espacios métricos separables.

\section{PRELIMINARES}

Sean $X$ e $Y$ espacios topológicos y consideremos el conjunto

$$
C(X, Y)=\{f ; f: X \rightarrow Y \text { es una función continua }\} \text {. }
$$

Si $X=Y$, el símbolo $i d_{X}$ significa que $i d_{X} \in C(X, X)$ es la función identidad.

\section{Definición 1.}

1) $1_{a} \in C(X, Y)$ significa que $1_{a}(x)=a \forall x \in X ; a \in Y$ (a fijo).

2) $A \subset X$ es un retracto de $X$ si existe $r \in C(X, A)$ tal que $r_{\triangle A}=i d_{A}$.

3) $f \in C(X, Y)$ es un homeomorfismo entre $X$ e $Y$, denotado por $f: X \approx Y$; si existe $g \in C(Y, X)$ tal que $f \circ g=i d_{Y}$ y $g \circ f=i d_{X}$. En este caso denotemos $f^{-1}=g, f^{-1}: X \approx Y$.

4) $X$ e $Y$ son espacios homeomorfos, denotado por $X \approx Y$; si existe $f \in C(X, Y)$ tal que $f: X \approx Y$.

1 Universidad Nacional Mayor de San Marcos - Facultad de Ciencias Matemáticas - Instituto de Investigación. 
5) Sean $f, g \in C(X, Y), f$ es homotópico a $g$, denotado por $f \simeq g$ si existe $h \in C(X \times \mathbb{I}, Y)$ tal que:

$$
h(x, 0)=f(x) \text { y } h(x, 1)=g(x) \quad \forall x \in X ; \mathbb{I}=[0,1] .
$$

6) $f \in C(X, Y)$ es una equivalencia homotópica; si existe $g \in C(Y, X)$ tal que $f \circ g \simeq i d_{Y}$ y $g \circ f \simeq i d_{X}$.

7) $X$ e $Y$ tienen el mismo tipo de homotopía; denotado por $X \simeq Y$; si existe una equivalencia homotópica $f \in C(X, Y)$.

8) $X$ es contráctil si existe $x \in X$ tal que $X \simeq\{x\}$.

9) Sea $I \neq \varnothing$ y para cada $\lambda \in I, X_{\lambda}$ un conjunto. El conjunto

$$
\prod_{\lambda \in I} X_{\lambda}=\left\{x: I \rightarrow \bigcup_{\lambda \in I} X_{\lambda} ; x(\lambda)=x_{\lambda} \in X_{\lambda}, \forall \lambda \in I\right\},
$$

se denomina "producto cartesiano de la familia $A_{\lambda}, \lambda \in I$ ".

10) Sean $f_{\lambda}: X_{\lambda} \rightarrow Y_{\lambda}$ un conjunto de funciones y sea una familia no vacía de conjuntos no vacíos $X_{\lambda}, Y_{\lambda}$. Para cada $\lambda \in I$, la función,

$$
f=\prod_{\lambda \in I} f_{\lambda}: \prod_{\lambda \in I} X \rightarrow \prod_{\lambda \in I} Y_{\lambda}
$$

dado por

$$
f\left(\left(x_{\lambda}\right)\right)=\left(f_{\lambda}\left(x_{\lambda}\right)\right), \forall\left(x_{\lambda}\right)_{\lambda \in I} \in \prod_{\lambda \in I} X_{\lambda}
$$

se denomina "función producto".

Observación.- Como consecuencia de la definición 1, tenemos que $X \simeq Y$ si $X \approx Y$.

Teorema 1. Las afirmaciones siguientes son equivalentes :

1) $X$ es contráctil

2) Existe $a \in X$ tal que $i d_{X} \simeq 1_{a}$.

Demostración. Si $X$ es contráctil, existe $a \in X$ tal que $X \simeq\{a\}$. Luego existe

$$
(f, g) \in C(X,\{a\}) \times C(\{a\}, X)
$$


tal que

$$
f \circ g \simeq i d_{\{a\}}, 1_{g(a)}=g \circ f \simeq i d_{X}
$$

de aquí existe $b=g(a) \in X$ tal que $i d_{X} \simeq 1_{b}$.

Inversamente, si existe $a \in X$ tal que, $i d_{X} \simeq 1_{a}, \quad i d_{X} \simeq 1_{a}=i \circ 1_{a} \quad$ y $1_{a} \circ i=1_{a} \simeq 1_{a}$; donde $i \in C(\{a\}, X)$ es la inclusión.

Luego $i \circ 1_{a} \simeq i d_{X}$ y $1_{a} \circ i \simeq 1_{a}$, existe $a \in X$ tal que $X \simeq\{a\}$.

Lema 1. Sean $f, \varphi \in C(X, Y)$ y $g, \psi \in C(Y, Z)$. Si $f \simeq \varphi$ y $g \simeq \psi$, entonces $f \circ g \simeq \varphi \circ \psi$.

Demostración. Si $f \simeq \varphi$ y $g \simeq \psi$, existe $(h, k) \in C(X \times \mathbb{I}, Y) \times C(Y \times \mathbb{I}, Z)$ tal que

$$
\begin{aligned}
& h(x, 0)=f(x), h(x, 1)=\varphi(x) \forall x \in X \quad \mathrm{y} \\
& k(y, 0)=g(y), k(y, 1)=\psi(y) \forall y \dot{E} Y .
\end{aligned}
$$

Sea $\Delta \in C(\mathbb{I}, \mathbb{I} \times \mathbb{I})$ la diagonal dada por

dado por

$$
\Delta(t)=(t, t) \forall t \in \mathbb{I} \text { y } r \in C(X \times(\mathbb{I} \times \mathbb{I}),(X \times \mathbb{I}) \times \mathbb{I})
$$

$$
r(x,(m, n))=((x, m), n), \quad \forall(x, m, n) \in X \times \mathbb{I} \times \mathbb{I} .
$$

Luego de los diagramas

$$
h: X \times \mathbb{I} \rightarrow Y, \quad k: Y \times \mathbb{I} \rightarrow Z \quad \text { y } \quad \Delta: \mathbb{I} \rightarrow \mathbb{I} \times \mathbb{I}, \quad i d_{X}: X \rightarrow X,
$$

tenemos el diagrama

$$
X \times \mathbb{I} \stackrel{i d_{X} \times \Delta}{\longrightarrow} X \times(\mathbb{I} \times \mathbb{I}) \stackrel{r}{\approx}(X \times \mathbb{I}) \times \mathbb{I} \stackrel{h \times i d_{\mathbb{I}}}{\longrightarrow} Y \times \mathbb{I} \stackrel{k}{\longrightarrow} Z
$$

Luego,

$$
\begin{gathered}
w=k \circ\left(h \times i d_{\mathbb{I}}\right) \circ r \circ\left(i d_{X} \times \Delta\right) \in C(X \times \mathbb{I}, Z) \mathrm{y} \\
w(x, t)=\left(k \circ\left(h \times i d_{\mathbb{I}}\right) \circ r \circ\left(i d_{X} \times \Delta\right)\right)(x, t)=k(h(x, t), t),
\end{gathered}
$$

entonces

$$
\begin{gathered}
w(x, 0)=k(h(x, 0), 0)=k(f(x), 0)=g(f(x))=g \circ f(x) \quad y \\
w((x, 1))=k(h(x, 1), 1)=k(\varphi(x), 0)=\psi(\varphi(x))=\psi \circ \varphi(x) .
\end{gathered}
$$

Por lo tanto, $f \circ g \simeq \varphi \circ \psi$. 
Proposición 1. Sea $(f, g) \in C(X, Y) \times C(Y, Z)$ con $f \circ g$ y $g$ equivalencias homotópicas, entonces, $f$ es una equivalencia homotópica.

Demostración. Si existe $(\varphi, \psi) \in C(Z, Y) \times C(Z, X)$ tal que,

$$
\varphi \circ g \simeq i d_{Y}, \quad g \circ \varphi \simeq i d_{Z} \quad \text { y } \psi \circ(g \circ f) \simeq i d_{X},(f \circ g) \circ \psi \simeq i d_{Z} .
$$

Luego,

$$
\begin{gathered}
(\psi \circ g) \circ f=\psi \circ(g \circ f) \simeq i d_{X} \text { y } \\
f \circ(\psi \circ g)=\left(i d_{Y} \circ f\right) \circ(\psi \circ g) \simeq((\varphi \circ g) \circ f) \circ(\psi \circ g)=(((\varphi \circ g) \circ f) \circ \psi) \circ g \\
=((\varphi \circ g) \circ(f \circ \psi)) \circ g=(\varphi \circ(g \circ(f \circ \psi))) \circ g \\
=(\varphi \circ((g \circ f) \circ \psi)) \circ g \simeq\left(\varphi \circ i d_{Z}\right) \circ g=\varphi \circ g \simeq i d_{Y} .
\end{gathered}
$$

Corolario 1. Sea $X \simeq Y$. Si $X$ es contráctil, $Y$ también lo es.

Demostración. Si $X$ es contráctil, existe $a \in X$ tal que $i d_{X} \simeq 1_{a}$ y como $X \simeq Y$, existe, tal que,

$$
f \circ g \simeq i d_{Y} \quad \text { y } \quad g \circ f \simeq i d_{X} .
$$

Pongamos $b=f(a)$ y del diagrama

$$
X \underset{1_{a}}{\stackrel{i d_{X}}{\longrightarrow}} X \underset{f}{\stackrel{f}{\longrightarrow}} Y
$$

tenemos que $f=f \circ i d_{X} \simeq f \circ 1_{a}=1_{b}$.

Luego del diagrama,

$$
Y \underset{g}{\stackrel{g}{\longrightarrow}} X \underset{1_{b}}{\stackrel{f}{\longrightarrow}} X
$$

tenemos que $i d_{Y} \simeq f \circ g \simeq 1_{b} \circ g=1_{b}$ entonces $Y$ es contráctil.

Corolario 2. Si $X \simeq Y$ y $Y \simeq Z$, entonces $X \simeq Z$.

Demostración. Si $X \simeq Y$ y $Y \simeq X$, existen

$$
(f, \varphi) \in C(X, Y) \times C(Y, X) \text { y }(g, \psi) \in C(Y, Z) \times C(Z, Y)
$$

tal que

$$
\varphi \circ f \simeq i d_{X}, f \circ \varphi \simeq i d_{Y} \quad \mathrm{y} \quad \psi \circ g \simeq i d_{Y}, g \circ \psi \simeq i d_{Z} .
$$

Luego el diagrama 




tenemos que

$$
\begin{aligned}
i d_{X}=i d_{X} \circ i d_{X} & \simeq(\varphi \circ f) \circ(\varphi \circ f)=\varphi \circ(f \circ \varphi) \circ f \\
& \simeq \varphi \circ(\psi \circ g) \circ f=(\varphi \circ \psi) \circ g \circ f \quad \mathrm{y} \\
i d_{Z}=i d_{Z} \circ i d_{Z} & \simeq(g \circ \psi) \circ(g \circ \psi)=g \circ(\psi \circ g) \circ \psi \\
& \simeq g \circ(f \circ \varphi) \circ \psi=g \circ f \circ(\varphi \circ \psi)
\end{aligned}
$$

Por tanto $X \simeq Z$.

Corolario 3. Sea $f \in C(X, Y)$. Si $X \circ Y$ es contráctil, entonces existe $b \in Y$ tal que $f \simeq 1_{b}$.

Demostración. Si $X$ es contráctil, existe $a \in X$ tal que $i d_{X} \simeq 1_{a}$.

Del diagrama

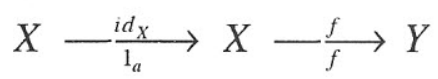

tenemos que

$$
f=f \circ i d_{X} \simeq f \circ 1_{a}=1_{a},
$$

existe $b=f(a) \in Y$ tal que $f \simeq 1_{b}$.

Si $Y$ es contráctil, existe $b \in Y$ tal que $i d_{Y} \simeq 1_{b}$. Del diagrama

$$
X \underset{f}{\stackrel{f}{\longrightarrow}} Y \underset{1_{b}}{\stackrel{i d_{Y}}{\longrightarrow}} Y
$$

tenemos que $f=i d_{Y} \circ f \simeq 1_{b} \circ f=1_{b}$, existe $b \in Y$ tal que $f \simeq 1_{b}$.

Proposición 2. Sean $1_{a}, 1_{b} \in C(X, Y)$, las relaciones siguientes son equivalentes:

1) $1_{a} \simeq 1_{b}$

2) a y b están en la misma componente conexa por caminos de $Y$.

Demostración. Si $1_{a} \simeq 1_{b}$, existe, tal que

$$
h(x, 0)=a \text { y } h(x, 1)=b \quad \forall x \in X .
$$


Luego $h \in C(\{x\} \times \mathbb{I}, Y)$ y como $f: \mathbb{I} \approx\{x\} \times \mathbb{I}$ dado por $f(t)=(x, t) \quad \forall t \in \mathbb{I}$, entonces existe $g=h \circ f \in C(\mathbb{I}, Y)$ tal que $g(0)=a$ y $g(1)=b$.

Inversamente, si existe $f \in C(\mathbb{I}, Y)$ tal que $f(0)=a$ y $f(1)=b$, definimos $h=f \circ p: X \times \mathbb{I} \rightarrow Y$; donde $p \in C(X \times \mathbb{I}, \mathbb{I})$ es la proyección en $\mathbb{I}$.

Luego

$$
h \in C(X \times \mathbb{I}, Y) \text { con } h(x, 0)=a \text { y } h(x, 1)=b \text {, entonces } 1_{a} \simeq 1_{b} .
$$

Corolario 4. Sean $f, g \in C(X, Y)$. Si $X$ es contráctil y $Y$ es conexo por caminos entonces $f \simeq g$.

Demostración. Si $X$ es contráctil, existen $a, b \in Y$ tal que $f \simeq 1_{a}, g \simeq 1_{b}$ y si $Y$ es conexo por caminos, $a$ y $b$ están en la misma componente conexa por conexos de $Y$. Luego, $f \simeq 1_{a} \simeq 1_{b} \simeq g$.

Teorema 2. Si $X$ es contráctil, entonces es conexo por caminos.

Demostración. Si $X$ es contráctil, existe $a \in X$ tal que $i d_{X} \simeq 1_{a}$. Luego existe $h \in C(X \times \mathbb{I}, X)$ tal que $h(x, 0)=i d_{X}(x)$ y $h(x, 1)=a \forall x \in X$.

Sea $x \in X, h \in C(\{x\} \times \mathbb{I}, X)$ y como $f: \mathbb{I} \approx\{x\} \times \mathbb{I}$ dado por $f(t)=(t, x) \forall t \in \mathbb{I}$, entonces

$$
\alpha_{x}=h \circ f \in C(\mathbb{I}, X) \text { y } \alpha_{x}(0)=x, \alpha_{x}(1)=a
$$

Sea $y \in X, \alpha_{y} \in C(\mathbb{I}, X)$ y $\alpha_{y}(0)=y, \alpha_{y}(1)=a$. Definamos, $\alpha: \mathbb{I} \rightarrow X$ por

$$
\alpha(t)=\left\{\begin{array}{cc}
\alpha_{x}(2 t) & , \quad 0 \leq t \leq \frac{1}{2} \\
\alpha_{y}(2-2 t), & \frac{1}{2} \leq t \leq 1
\end{array}\right.
$$

Para $t=\frac{1}{2}, \alpha_{x}\left(2\left(\frac{1}{2}\right)\right)=\alpha_{x}(1)=a=\alpha_{y}\left(2-2\left(\frac{1}{2}\right)\right)$, entonces

$$
\alpha \in C(\mathbb{I}, X) \text { y } \alpha(0)=x, \alpha(1)=y \text {. }
$$

Por lo tanto, $X$ es conexo por caminos.

Corolario 5. Sea $a \in X$. Si $X$ es contráctil, entonces $i d_{X}=1_{a}$. 
Corolario 6. Sean $f, g \in C(X, Y)$. Si $Y$ es contráctil, entonces $f \simeq g$.

Demostración. Si $Y$ es contráctil, existe $b \in Y$ tal que $f \simeq 1_{b}$.

Del diagrama

$$
X \underset{g}{\stackrel{g}{\longrightarrow}} Y \underset{1_{b}}{\stackrel{i d_{y}}{\longrightarrow}} Y
$$

tenemos que $g=i d_{Y} \circ g \simeq 1_{b} \circ g=1_{b} \simeq f$.

Proposición 3. Sea $\left(X_{\lambda}\right)_{\lambda \in L}$ una familia de espacios topológicos. Entonces

$$
X=\prod_{\lambda \in L} X_{\lambda} \simeq \prod_{\lambda \in L} Y_{\lambda}=Y, \text { siempre que } X_{\lambda} \simeq Y_{\lambda} \forall \lambda \in L
$$

Demostración. Sea $\lambda \in L$ con $X_{\lambda} \simeq Y_{\lambda}$, existe

$$
\left(f_{\lambda}, g_{\lambda}\right) \in C\left(X_{\lambda}, Y_{\lambda}\right) \times C\left(Y_{\lambda}, X_{\lambda}\right)
$$

tal que

$$
f_{\lambda} \circ g_{\lambda} \simeq i d_{Y_{\lambda}} \text { y } \quad g_{\lambda} \circ f_{\lambda} \simeq i d_{X_{\lambda}}
$$

Luego existe

$$
h_{\lambda} \in C\left(X_{\lambda} \times \mathbb{I}, X_{\lambda}\right)
$$

tal que $h_{\lambda}(x, 0)=x$ y $h_{\lambda}(x, 1)=g_{\lambda} \circ f_{\lambda}(x) \forall x \in X_{\lambda}$, entonces,

$$
\prod_{\lambda \in L}\left(h_{\lambda}\right) \in C\left(\prod_{\lambda \in L}\left(X_{\lambda} \times \mathbb{I}\right), \prod_{\lambda \in L} X_{\lambda}\right) .
$$

Definimamos

$$
\begin{gathered}
\Delta: \mathbb{I} \rightarrow \mathbb{I}^{L} \text { por } \Delta(t)=\left(t_{\lambda}\right)_{\lambda \in L} \forall t \in \mathbb{I} ; t_{\lambda}=t \forall \lambda \in L \\
f:\left(\prod_{\lambda \in L} X_{\lambda}\right) \times \mathbb{I}^{L} \approx \prod_{\lambda \in L}\left(X_{\lambda} \times \mathbb{I}\right)
\end{gathered}
$$

dada por

$$
\begin{gathered}
f\left(\left(a_{\lambda}\right)_{\lambda \in L},\left(r_{\lambda}\right)_{\lambda \in L}\right)=\left(\left(a_{\lambda}, r_{\lambda}\right)_{\lambda \in L}\right) \forall\left(\left(a_{\lambda}\right)_{\lambda \in L},\left(r_{\lambda}\right)_{\lambda \in L}\right) \in\left(\prod_{\lambda \in L} X_{\lambda}\right) \times \mathbb{I}^{L} \quad \mathrm{y} \\
\left(i d_{X} \times \Delta\right): X \times \mathbb{I} \rightarrow X \times \mathbb{I}^{L}
\end{gathered}
$$


dado por

$$
\left(i d_{X} \times \Delta\right)(x, t)=\left(x,\left(t_{\lambda}\right)_{\lambda \in L}\right) \forall(x, t) \in X \times \mathbb{I} .
$$

Del diagrama

$$
X \times \mathbb{I} \stackrel{\left(i d_{X} \times \Delta\right)}{\longrightarrow} X \times \mathbb{I}^{L} \stackrel{f}{\approx} \prod_{\lambda \in L}\left(X_{\lambda} \times \mathbb{I}\right) \stackrel{\prod_{\lambda \in L} h_{\lambda}}{\longrightarrow} X
$$

tenemos

$$
\begin{gathered}
h=\left(\prod_{\lambda \in L} h_{\lambda}\right) \circ f \circ\left(i d_{X} \times \Delta\right) \in C(X \times \mathbb{I}, X) \quad \text { y } \\
h\left(\left(a_{\lambda}\right)_{\lambda \in L}, 0\right)=\left(\prod_{\lambda \in L} h_{\lambda}\right) \circ f\left(\prod_{\lambda \in L} a_{\lambda}, \prod_{\lambda \in L} 0_{\lambda}\right)=\left(\prod_{\lambda \in L} h_{\lambda}\left(a_{\lambda}, 0\right)\right)=\left(a_{\lambda}\right)_{\lambda \in L,}, \\
h\left(\left(a_{\lambda}\right)_{\lambda \in L}, 1\right)=\left(\prod_{\lambda \in L} h_{\lambda}\right) \circ f\left(\left(a_{\lambda}\right)_{\lambda \in L},\left(1_{\lambda}\right)_{\lambda \in L}\right)=\prod_{\lambda \in L} h_{\lambda}\left(a_{\lambda}, 1\right)= \\
\prod_{\lambda \in L}\left(g_{\lambda} \circ f_{\lambda}\right)\left(a_{\lambda}\right)=\left(\prod_{\lambda \in L} g_{\lambda}\right) \circ\left(\prod_{\lambda \in L} f_{\lambda}\right)\left(\left(a_{\lambda}\right)_{\lambda \in L}\right) .
\end{gathered}
$$

Luego

$$
\left(\prod_{\lambda \in L} g_{\lambda} g_{\lambda}\right) \circ\left(\prod_{\lambda \in L} f_{\lambda} f_{\lambda}\right) \simeq i d_{X} .
$$

Análogamente se tiene que

$$
\left(\prod_{\lambda \in L} f_{\lambda}\right) \circ\left(\prod_{\lambda \in L} g_{\lambda}\right) \simeq i d_{Y}
$$

Por lo tanto, $X \simeq Y$.

Lema 2. Si $X$ es contráctil, entonces $X \times Y \simeq Y$.

Demostración. Si $X$ es contráctil, existe $a \in X$ tal que $X \simeq\{a\}$. Luego $X \times Y \simeq\{a\} \times Y \quad$ y como $\{a\} \times Y \simeq Y$ (ya que $\{a\} \times Y \approx Y$ ), por lo tanto, $X \times Y \simeq Y$.

Teorema 3. Sea $\left(X_{\lambda}\right)_{\lambda \in L}$ una familia de espacios topológicos. Son equivalentes: 
1) $X=\prod_{\lambda \in L} X_{\lambda}$ contráctil

2) $X_{\lambda}$ es contráctil $\forall \lambda \in L$.

Demostración. Sea $a_{\beta} \in X_{\beta}$ y para $\lambda \neq \beta$ en $L$, definamos $i_{\lambda}: X_{\lambda} \rightarrow X$ por $i_{\lambda}\left(x_{\lambda}\right)=\left(y_{\lambda}\right)_{\alpha \in L} ;$

$$
y_{\alpha}= \begin{cases}a_{\beta} & , \quad \alpha \neq \lambda \\ x_{\beta} & , \quad \alpha=\lambda\end{cases}
$$

Sea $p_{\lambda}: X \rightarrow X_{\lambda}$ la proyección y $\left(i_{\lambda} \circ i d_{\mathbb{I}}\right): X_{\lambda} \times \mathbb{I} \rightarrow X \times \mathbb{I}$ dado por

$$
\left(i_{\lambda} \circ i d_{\mathbb{I}}\right)(x, t)=\left(i_{\lambda}(x), t\right) \quad \forall(x, t) \in X_{\lambda} \times \mathbb{I} .
$$

Si $X$ es contráctil, existe $b \in X$ tal que $i d_{X} \simeq 1_{b}$. Luego, existe

$$
h \in C(X \times \mathbb{I}, X) \quad \text { tal que } h(x, 0)=x \text { y } \dot{h(x, 1)}=b \quad \forall x \in X .
$$

Del diagrama

$$
X_{\lambda} \times \mathbb{I} \stackrel{\left(i_{\lambda} \times i d_{\mathbb{I}}\right)}{\longrightarrow} X \times \mathbb{I} \stackrel{h}{\longrightarrow} X \stackrel{p_{\lambda}}{\longrightarrow} X_{\lambda}
$$

tenemos

$$
\begin{gathered}
k=p_{\lambda} \circ h \circ\left(i_{\lambda} \times i d_{\mathbb{I}}\right) \in C\left(X_{\lambda} \times \mathbb{I}, X_{\lambda}\right) \quad y \\
k\left(x_{\lambda}, 0\right)=p_{\lambda} \circ h\left(i_{\lambda}\left(x_{\lambda}\right), 0\right)=p_{\lambda} \circ i_{\lambda}\left(x_{\lambda}\right)=x_{\lambda}, k\left(x_{\lambda}, 1\right)= \\
=p_{\lambda} \circ h \circ\left(i_{\lambda}\left(x_{\lambda}\right), 1\right)=p_{\lambda}(b)=c \quad \forall x_{\lambda} \in X_{\lambda} .
\end{gathered}
$$

Luego existe $c \in X_{\lambda}$ tal que $i d_{X_{i}} \simeq 1_{c}$, entonces $X_{\lambda}$ es contráctil. Inversamente, sea $\lambda \in L$ con $X_{\lambda}$ contráctil, existe $b_{\lambda} \in X$ tal que $X_{\lambda} \simeq\left\{b_{\lambda}\right\}$. Entonces $X=\prod_{\lambda \in L} X_{\lambda} \simeq \prod_{\lambda \in L}\left\{b_{\lambda}\right\}$ y como $\prod\left\{b_{\lambda}\right\}=\left\{\left(b_{\lambda}\right)_{\lambda \in L}\right\}$, se sigue que $X$ es contráctil.

Proposición 4. Las afirmaciones siguientes son equivalentes :

1) $A$ es un retracto de $X$

2) Existe $r \in C(X, X)$ tal que $r \circ r=r$ y $A=r(X)$.

Demostración. Si $A$ es un retracto de $X$, existe $r \in C(X, A)$ tal que $\Upsilon_{A}=i d_{A}$. Luego $r \circ r=r$ y $A=r(X)$. Inversamente, si $r \in C(X, X)$ tal que $r \circ r=r$ y $A=r(X)$, entonces existe $r \in C(X, X)$ tal que $r_{A}=i d_{A}$. 
Teorema 4. Sea A un retracto de $X$. Entonces:

1) A es un cerrado si $X$ es de Hausdorff.

2) A es conexo si $X$ lo es.

3) A es localmente conexo si $X$ lo es.

4) A es localmente conexo por caminos si $X$ lo es.

5) A es conexo por caminos si $X$ lo es.

6) A es contráctil si $X$ lo es.

Demostración. Sea $r \in C(X, A)$ la retracción.

(1) $A=r(X)=\{y \in X ; \quad r(y)=y\}$ es cerrado ya que $X$ es de Hausdorff.

(2) Es obvio.

(3) Sea $a \in A$ y $V \in v_{A}(a)$, por la continuidad de $r$, existe $W \in v_{X}(a)$ tal que $r(W) \subset V$ y por la hipótesis, existe $M \in v_{X}(a)$ conexo tal que $M \subset W$. Luego, $a \in r(W) \subset V$ y $r(M)$ es conexo. Como

$$
M \cap A \in v_{A}(a) \text { y } M \cap A=r(M \cap A) \subset r(M), r(M) \in v_{A}(a) .
$$

Por lo tanto $A$ es localmente conexo.

4) Sea $a \in A$ y $V \in v_{A}(a)$, por la continuidad de $r$ existe $W \in v_{X}(a)$ tal que $r(W) \subset V$ y por hipótesis, existe $M \in v_{X}(a)$ conexo por caminos tal que $M \subset V$, luego $a \in r(W) \subset V$ y $r(M)$ es conexo. Como

$$
M \cap A \in v_{A}(a) \quad \text { y } \quad M \cap A=r(M \cap A) \subset r(M), r(M) \in v_{A}(a)
$$

es conexo por caminos. Por lo tanto, $A$ es localmente conexo por caminos.

5) Sea $f \in C(\{0,1\}, A)$ y como $X$ es localmente conexo por caminos, existe $g \in C(\mathbb{I}, X)$ tal que $g_{\{\{0,1\}}=f$. Tomando $h=r \circ g \in C(\mathbb{I}, A), h_{\{0,1\}}=f$.

Por lo tanto $A$ es conexo por caminos.

6) Si $X$ es contráctil, existe $a \in X$ tal que $i d_{X} \simeq 1_{a}$ y como $A$ es un retracto de $X$ existe $r \in C(A, X)$ tal que $r_{A}=i d_{A}$. Pongamos $i: A \rightarrow X$ la conclusión y del diagrama

$$
A \underset{i}{\stackrel{i}{\longrightarrow}} X \underset{1_{a}}{\stackrel{i d_{X}}{\longrightarrow}} X \underset{r}{\stackrel{r}{\longrightarrow}} A
$$


tenemos que $i d_{A}=r \circ i=r \circ i d_{X} \circ i \simeq r \circ 1_{a} \circ i=1_{a}$, entonces $A$ es contractil.

Proposición 5. Sea $X=A \cup B$ con $A \cap B=\{p\}$. Si $A$ y $B$ son cerrados, entonces $A$ y $B$ son retractos de $X$.

Demostración. Si $A$ y $B$ son cerrados, definamos $f: X \rightarrow A$ por

$$
f=\left\{\begin{array}{lll}
x & \text { si } & x \in A \\
p & \text { si } & x \in B
\end{array}\right.
$$

Dado que $i d_{A}$ y $1_{p}$ coinciden en $A \cap B=\{p\}, f \in C(X, A)$ y $f_{\backslash_{A}}=i d_{A}$. Luego $A$ es un retracto de $X$. Haciendo $B=A$, se demuestra que $B$ es un retracto de $X$.

Proposición 6. Sean $A \subset X$ y $Y \neq \varnothing$. Las afirmaciones siguientes son equivalentes :

1) A es un retracto de $X$.

2) $A \times Y$ es un retracto de $X \times Y$.

Demostración. Denotemos $i: A \rightarrow X, j: A \times Y \rightarrow X \times Y$ las inclusiones. Además, para $b \in Y$, definamos $g: X \rightarrow X \times Y$ dado por $g(x)=(x, b) \forall x \in X \quad \mathrm{y}$ $p: X \times A \rightarrow A$ la proyección en $A$.

Si $A$ es un retracto de $X$, existe $r \in C(X, A)$ tal que $r \circ i=i d_{A}$. Luego $\left(i \times i d_{X}, \quad r \times i d_{Y}\right) \in C(A \times X, X \times Y) \times C(Y \times X, A \times Y)$ y $\left(r \times i d_{Y}\right) \circ i \times i d_{X}$, AxY es un retracto de $X \times Y$. Inversamente, si $A \times Y$ es un retracto de $X \times Y$, existe $r \in C(X \times Y, A \times X)$ tal que $r \circ j=i d_{A \times Y}$. Luego

$$
\begin{gathered}
\left(p \circ i d_{A \times Y} \circ g\right)(a)=p\left(i d_{A \times Y}(g(a))\right)=p\left(i d_{A \times Y}(a, b)\right)=a \forall a \in A \quad \text { y } \\
(p \circ r \circ g)(a)=p \circ r(g(a))=p(r(a, b))=p(a, b)=a \quad \forall a \in A .
\end{gathered}
$$

Entonces

$$
(p \circ r \circ g) \in C(X, A) \quad \text { y } \quad p \circ r \circ g \circ i=i d_{A}
$$

lo que demuestra que $A$ es un retracto de $X$.

Proposición 7. Sean $A \subset B$ y $B \subset X$. Si $A$ es un retracto de $B$ y $B$ es un retracto de $X, A$ es un retracto de $X$. 


\section{ESPACIOS SÓlIDOS}

Decimos que un espacio de Hausdorff $Y$ es "sólido", si para cada subconjunto cerrado $A$ de un espacio normal de Hausdorff $X \quad$ y $\forall f \in C(A, Y)$, existe $g \in C(X, Y)$ tal que $g_{\backslash_{A}}=f$.

El siguiente es un resultado clásico para la topología de conjuntos.

\section{Teorema de Tietze}

Sea $X$ un espacio de Hausdorff. Las afirmaciones siguientes son equivalentes :

1) $X$ es normal

2) $\forall A \subset X$ cerrado y $\forall f \in C(A, \mathbb{I})$, existe $g \in C(X, \mathbb{I})$ tal que $g_{\backslash_{A}}=f$.

\section{Ejemplos de espacios sólidos}

Por el teorema de Tietze, II es sólido y por ([1]; pags. 134 - 135) se tiene que $(-1,1)$ y $(-1,1]$ son sólidos.

\section{Observación.}

1) Como consecuencia de la definición, tenemos que la solidez es invariante bajo homeomorfismo.

2) $\mathbb{R}$ y los intervalos en $\mathbb{R}$ son espacios sólidos..

Proposición 8. Sea $\left(X_{i}\right)_{i \in I}$ una familia de espacios topológicos. Las afirmaciones siguientes son equivalentes :

1) $X=\prod_{i \in I} X_{i}$ es sólido

2) Para cada $i \in I, X_{i}$ es sólido.

Demostración. Sea $a_{\beta} \in X_{\beta}$ y para $\lambda \neq \beta$ en I, definamos $i_{\lambda}: X_{\lambda} \rightarrow X$ por $i_{\lambda}\left(x_{\lambda}\right)=\left(y_{\lambda}\right)_{\alpha \in L} ;$

$$
y_{\alpha}= \begin{cases}a_{\beta}, & \alpha \neq \lambda \\ x_{\lambda}, & \alpha=\lambda\end{cases}
$$

Sea $p_{\lambda}: X \rightarrow X_{\lambda}$ la proyección, $p_{\lambda} \circ i_{\lambda}=i d_{x_{\lambda}}$. Sea $A$ un subconjunto cerrado de un espacio de Hausdorff normal $Y$ y $i: A \rightarrow Y$ la inclusión. Si 
$f \in C\left(A, X_{\lambda}\right)$ y $X$ es sólido, existe $g \in C(Y, X)$ tal que $g \circ i=i_{\lambda} \circ f$.

Entonces existe $h=p_{\lambda} \circ g \in C\left(Y, X_{\lambda}\right)$ tal que $h \circ i=p_{\lambda} \circ g \circ i=p_{\lambda} \circ i_{\lambda} \circ f$ $=i d_{x_{\lambda}} \circ f=f$. Por lo tanto, $X_{\lambda}$ es sólido; $\forall \lambda \in I$. Inversamente, si $X_{\lambda}$ es sólido y si $f \in C(A, X), \exists_{g_{\lambda}} \in C\left(Y, X_{\lambda}\right)$ tal que $g_{\lambda} \circ i=p_{\lambda} \circ f$. Luego, existe $\prod_{i \in I} g_{\lambda} \in C(Y, X)$ tal que

$$
\left(\prod_{i \in I} g_{\lambda}\right) \circ i=\left(\prod_{i \in I}\left(g_{\lambda} \circ i\right)\right)=\prod_{i \in I}\left(p_{\lambda} \circ f\right)=f
$$

pues, $a \in A$ y $f(a)=\left(f_{\lambda}(a)\right)_{i \in I}, \quad p_{\lambda}(f(a))=f_{\lambda}(a)$. De aquí

$$
\left(\prod_{i \in I}\left(p_{\lambda} \circ f\right)\right)(a)=\prod_{i \in I}\left(p_{\lambda}(f(a))\right)=\prod_{i \in I}\left(f_{\lambda}(a)\right)=\left(f_{\lambda}(a)\right)_{i \in I}=f(a) .
$$

Luego existe $h=\prod_{i \in I} g_{\lambda} \in C(Y, X)$ tal que $h \circ i=\prod\left(p_{\lambda} \circ f\right)=f$, entonces $X$ es sólido.

Corolario 7. El $n$-cubo unidad, $\mathbb{I}^{n}$, y el $n$-espacio euclideano, $\mathbb{R}^{n}$, y sus interiores son espacios sólidos.

Teorema 5. Sea A un subconjunto de un espacio normal sólido X. Las afirmaciones siguientes son equivalentes:

1) $A$ es un retracto de $X$

2) $A$ es sólido y cerrado.

Demostración. Si $A$ es un retracto de $X$, existe $r \in C(X, A)$ tal que $\tau_{A}=i d_{A} y$ $A$ es cerrado ( $X$ es sólido).

Sea $B$ un subconjunto de un espacio normal $Y$ y $f \in C(B, A)$.

Sea $(i, j) \in C(B, Y) \times C(A, X)$ las inclusiones y como $X$ es sólido, existe $h \in C(Y, X)$ tal que $j \circ f=h \circ i$.

Tomando $\varphi=i d_{A} \circ r \circ h \in C(B, A)$, entonces $\varphi \circ i=f$. Inversamente, si $A$ es sólido y cerrado, existe $r \in C(X, A)$ tal que $\kappa_{A}=i d_{A}$. Por lo tanto, $A$ es un retracto de $X$.

Corolario 8. La n-esfera, $\mathbb{S}^{n}$ no es sólido. 
Proposición 9. Sea $A$ un subconjunto de un espacio de Hausdorff $X$. Si $A$ es cerrado y sólido, entonces $A$ es un retracto de $X$.

Demostración. Es la recíproca del teorema anterior.

Proposición 10. Si X es sólido, entonces X es conexo por caminos.

Demostración. Sean $a, b \in X$, definamos $f:\{0,1\} \rightarrow X$ por $f(0)=a, f(1)=b$ y si $X$ es sólido, existe $g \in C(\mathbb{I}, X)$ tal que $g_{\backslash\{0,1\}}=f$. Por lo tanto, $X$ es conexo por caminos.

Corolario 9. Sean $f, g \in C(X, Y)$. Si $X$ es binormal y $Y$ es sólido, entonces $f \simeq g$.

Demostración. Sea $M=X \times\{0\} \bigcup X \times\{1\}$, definamos $k: M \rightarrow Y$ por

$$
k(x, t)=\left\{\begin{array}{ll}
f(x), & t=0 \\
g(x), & t=1
\end{array}\right. \text {. }
$$

y como $Y$ es sólido, existe $h \in C(X \times \mathbb{I}, Y)$ tal que $h_{M}=k$. Por lo tanto $f \simeq g$.

Teorema 6. $X$ es localmente conexo por caminos si $X$ es sólido compacto.

Demostración. Si $X$ es sólido compacto y por ([2]; 7, 118), $X$ se identifica a un subespacio de un cubo $\mathbb{I}^{L}$, es decir, existe $A \subset \mathbb{I}^{L}$ tal que $f: X \approx A$. Como $X$ es sólido, $A$ es sólido compacto y de aquí $A$ es un retracto de $\mathbb{I}^{L}$, entonces $A$ es localmente conexo por caminos. Por lo tanto $X$ es localmente conexo por caminos.

Teorema 7. $X$ es contráctil si es sólido binormal $(X \times \mathbb{I}$ es normal y $X$ es paracompacto).

Demostración. Sea $M \subset X \times \mathbb{I}$ y $a \in X$ con $M=X \times\{0,1\} \cup\{a\} \times \mathbb{I}$, defina$\operatorname{mos} f: M \rightarrow X$ por

$$
f(x, t)= \begin{cases}x, & t=0 \\ a, & x=a \\ . a & , \quad t=1\end{cases}
$$

Si $X$ es sólido, existe $g \in C(X \times \mathbb{I}, X)$ tal que $g_{\backslash_{M}}=f$ y de aquí existe $a \in X$ tal que $g \simeq 1_{a}$. Por lo tanto $X$ es contráctil. 
Proposición 11. Todo espacio sólido paracompacto es sólido binormal.

Demostración. Sea $X$ un espacio sólido paracompacto y por ([3]; x.1.12, 20), ([4]; 2.2; 163) se tiene que $X \times \mathbb{I}$ es normal paracompacto y se concluye del teore$\mathrm{ma}$ anterior.

Corolario 10. Todo espacio sólido paracompacto es contráctil.

Demostraremos simultáneamente que el espacio proyectivo $P_{n}$ sobre el conjunto de los números reales $\mathbb{R}$, los números complejos $\mathbb{C}$ y los cuaterniones $\mathbb{H}$ no son espacios sólidos.

Proposición 12. $P_{n} F$ no es un espacio sólido.

Demostración. Por ([5]; vii, 9.8, 224), tenemos que para $n>k>0, P_{k} F$ no es retracto de $P_{n} F$ y se concluye de la proposición 2.2.

\section{Unicoherencias en Espacios Sólidos}

Cerramos en esta parte con una implicación entre la «solidez» y la «unicoherencia» en espacios normales y localmente conexos por caminos. Pero la recíproca no es válida. Por ejemplo $\mathbb{C} P^{n}, n$-espacio proyectivo complejo y $\mathbb{R} P^{n}, n$-espacio proyectivo real $(n \geq 2)$ son normales, localmente conexos por caminos, unicoherentes $([6] ;)$ y no son sólidos.

Denotemos el plano complejo por $\mathbb{C}$, el plano complejo sin el origen por $\mathbb{C}^{*}$, y la función exponencial compleja por $e: \mathbb{C} \rightarrow \mathbb{C} *$. Escribiremos $\mathcal{F}(X)=C\left(X, \mathbb{C}^{*}\right)$ y para $f, g \in \mathcal{F}(X)$, definimos

$$
\begin{aligned}
(f \cdot g)(x) & =f(x) \cdot g(x), \\
(f / g)(x) & =f(x) / g(x), \\
1(x) & =1,
\end{aligned}
$$

para cada $x \in X$. Así, $\mathcal{F}(X)$ se convierte en un grupo abeliano. Escribiremos

$$
\varepsilon(X)=\left\{f \in \mathcal{F}(X) \text {; existe } \varphi \in C(X, \mathbb{C}) \text { tal que } f(x)=e^{\varphi(x)} \forall x \in X\right\} .
$$

Se ve que $\varepsilon(X)$ es un subgrupo abeliano de $\mathcal{F}(X)$.

Al grupo cociente

$$
B(X)=\mathcal{F}(X) / \varepsilon(X)
$$


se le denomina el grupo de $\underline{\text { Bruschlinsky }}$ de $X$. Además, $B(X) \simeq 1$ significa que $\mathcal{F}(X)=\varepsilon(X)$.

Proposición 13. Si $X$ es contráctil, entonces $B(X) \simeq 1$. Ver ([6]; II.1.23,20)

Sea $X$ un espacio conexo. Se dice que $X$ es un espacio unicoherente si para cada par de cerrados conexos $A, B$ tal que $X=A \cup B$ se tiene que $A \cap B$ es conexo.

Teorema 7. Sea $X$ un espacio conexo. Si $X$ es normal $y B(X) \simeq 1$ entonces $X$ es unicoherente. $\operatorname{Ver}([6] ;)$.

Proposición 14. Todo espacio sólido paracompacto es unicoherente.

Demostración. Si $X$ es sólido paracompacto entonces $X$ es normal, conexo y contráctil. Se sigue de proposición 13 y teorema 7 que $X$ es unicoherente.

Corolario 10. Sean $f, g \in C(X, Y)$. Si $X$ es contráctil y $Y$ es conexo por caminos, $f \simeq g \simeq 1_{b} \quad \forall b \in Y$.

Proposición 15. X es simplemente conexo si es sólido.

Demostración. Sean $\alpha, \beta \in C(\mathbb{I}, X)$ con $\alpha(0)=\alpha(1)=b=\beta(0)=\beta(1)$ y si $\quad X$ es sólido, $X$ es conexo por caminos y como $\mathbb{I}$ es contráctil, se tiene que $\alpha \simeq \beta \simeq 1_{b} \simeq 1_{a}, \forall a \in X$.

Teorema 8. Sea $Z$ un espacio localmente conexo por caminos y $p \in C(Y, X)$ un recubridor universal. Si $f \in C(Z, X)$ y $Z$ es simplemente conexo, existe $g \in C(Z, Y)$ tal que $p \circ g=f$. ([7])

Corolario 11. Sea X un espacio normal. Si $X$ es localmente conexo por caminos y simplemente conexa, entonces es unicoherente.

Demostración. Sea $f \in \mathcal{F}(X)$ y como la función exponencial compleja $e: \mathbb{C} \rightarrow \mathbb{C}^{*}$ es un recubridor, existe $\varphi \in C(X, \mathbb{C})$ tal que $f(x)=e^{\varphi(x)}, \forall x \in X$ De aqui $f \in \varepsilon(X)$ entonces $\mathcal{F}(X)=\varepsilon(X)$, y por teorema 8 , se tiene que $X$ es unicoherente.

Corolario 12. Sea X un espacio normal y localmente conexo por caminos. Si X es. sólido entonces $X$ es unicoherente. 
Finalmente, mencionamos para espacios métricos separables una relación entre espacios sólidos y los espacios retractos absolutos.

Un espacio metrizable $Y$ es un retracto absoluto si para todo espacio metrizable $E$ que contenga a $\mathrm{Y}$ como subespacio cerrado, $\mathrm{Y}$ es un retracto de $\mathrm{E}$.

Proposición 16. Sea Y un espacio métrico separable. Las afirmaciones siguientes son equivalentes ( Ricabarra [8]. v.7.21; 235):

1. Y es sólido

2. $Y$ es un retracto absoluto y $G_{g}$ absoluto.

\section{BIBLIOGRAFIA}

[1] Ayala Gómez, Rafael. Elementos de topología, Addison -Wesley, España, (1997).

[2] Kelley, J.L . Topología General, Argentina, Eudeba, (1962).

[3] Margalet Roig, J. Topología ****. Editorial Alhambra, España, (1980).

[4] Dugundi, J. Topología, Allyn and Bacon, (1996).

[5] Dold, A . Lecture on Algebraic Topology, Berlin-Heideberg, New York, Springer-Verlag, (1972).

[6] Olano Díaz, William C. Segundo Teorema Fundamental de Eilenberg. Tesis para optar título de Licenciado en Matemática, UNMSM, Lima-Perú, (2002).

[7] Lages Lima, Elon; Grupo Fundamental e Espaços de Recobrimento, IMPABrasil, (1993).

[8] Riacabarra, R.A., Larotonda, A.R. Notas sobre Topología Algebraica. 